\title{
A New Approach on Quantum Gravity in Lyra Geometry
}

\author{
Nilo Sylvio Costa Serpa ${ }^{1,2,3}$ \\ 1. GAUGE-F Scientific Researches, Brazil \\ 2. Institute of Science and Technology, Universidade Paulista, Brazil \\ 3. UNICESP, Brazil
}

\begin{abstract}
This article investigates quantum gravity through a new approach based on quantization of spacetime and Lyra geometry. Singularity functions are applied to the study, whose main focus is to investigate the physics in the surroundings of supermassive bodies. Present work is a continuation of the research program on quantum gravity and time machines established by the author in a previous publication. The physical and geometrical features of the model are discussed.
\end{abstract}

Key words: Singularity functions, Lyra's geometry, affine connection, gauge, Planck scale, parallel transfer.

\section{Nomenclature}

G-Closure: A bubble of compressed spacetime

$D$ : $\quad$ Covariant derivative

\section{Greek letters}

$\begin{array}{ll}\chi: & \text { Lyra's gauge function } \\ \Gamma_{\mu \sigma}^{\alpha}: & \text { Levi-Civita-Christofell connection } \\ { }^{\dagger} \Gamma_{\mu \sigma}^{\alpha}: & \text { Lyra's connection } \\ \phi_{\sigma}: & \text { Displacement vector field } \\ g_{\mu \sigma}: & \text { Tensor metric }\end{array}$

\section{Introduction}

In recent times, inspired by works of Rovelli [1-5] and Baez [6], and by some old profound considerations from Butterfield and Isham [7], I developed a new framework for quantum gravity in which, instead of thinking about gravity as consisting of supersymmetric particles, I imagined a quantum structure of the spacetime itself, meeting the idea that general relativity is, first of all, a theory about the geometry of spacetime, and the gravitational field a representation of the deformations of that geometry. For this purpose, I have adopted an analytical model of the nature of the geodesic paths in a gravitational field, defining the

Corresponding author: Nilo Sylvio Costa Serpa, Ph.D., professor, research fields: quantum gravity, quantum computing, cosmology and thermal systems engineering. invariant element of expansion or contraction of the Universe in commoving coordinates as the elementary "grumo" of spacetime. From this new perspective, I focused, in particular, on investigating the deformations that would lead time effects only, since time, as discussed previously [8], is the predominant dimension in the gravitational evolution of the heavenly systems. The suggested "granular" structure of the spacetime, that is, the size and type of the elementary "quanta of spacetime", was treated mathematically with the aid of the so-called "singularity functions" [8].

\section{The Quantum Spacetime Phenomenology}

The phenomenological model I chose to develop the construct of quantum spacetime was the interaction of two supermassive bodies with strains conflicting with one another. I considered singularities caused by spherical pressure from the outside in, not caused by a central high density; thus, unlike a hypothetical single central source, we have now several virtual external sources for each central singularity. Theoretically, such "bubbles" of compression can be generated by gravitational pressure caused by supermassive bodies close to each other like two black holes or two pulsars in close binary systems. Since bodies are not perfectly rigid, when two bodies interact gravitationally with one 
another their facing surfaces are more distorted because they are attracted more strongly than the opposite sides. But, the gravitational attraction between the bodies also tends to squeeze or compress them as they pull one another toward their center points; so, the strong attraction between massive bodies leads to a compression of space contained among them. This "bubble" of compressed space I called "G-closure" [8]. Therefore, gravitational "bubbles" of spacetime shrinkage within an expanding universe are plausible, featuring local inhomogeneities. We can speculate on the spacetime behavior in such physical conditions from the study of those supermassive binary systems.

\section{The Essential Lyra Geometry}

Many works appeared on cosmology applying Lyra geometry from authors as Reddy and Innaiah [9], Reddy and Venkateswarlu [10], both in the eighties, and more recently Shchigolev [11]. Shchigolev even says that "[...] Lyra's geometry can be considered as the candidate for modification of the contemporary cosmological models, the necessity of which is almost generally recognized" [11].

To fully understand the meaning of Lyra geometry, it is necessary to establish some preliminaries in GR (general relativity). In the context of GR, if we think about rigid motions in spacetime, we see that these motions are in fact gauge transformations, as it can be confirmed by the parallel transport of a vector given from Christoffel connection, say

$$
d v^{\alpha}=-v^{\mu} \Gamma_{\mu \sigma}^{\alpha} d v^{\sigma}
$$

or

$$
d v_{\mu}=v_{\alpha} \Gamma_{\mu \sigma}^{\alpha} d v^{\sigma}
$$

From here, once that the scalar product of two vectors at the same point is

we have

$$
\begin{gathered}
u . v=g_{\mu v} u^{\mu} v^{v} \therefore v \cdot v=g_{\mu v} v^{\mu} v^{v} \\
=|v|^{2}=\left(v_{v}, v^{v}\right)
\end{gathered}
$$

$$
\begin{aligned}
& d|v|^{2}=d\left(v_{v}, v^{v}\right)=d v_{v} v^{v}+v_{v} d v^{v} \\
& =v_{\alpha} \Gamma_{\mu \sigma}^{\alpha} d v^{\mu} v^{\sigma}-v_{v} v^{\mu} \Gamma_{\mu \alpha}^{v} d v^{\alpha}=0 .
\end{aligned}
$$

Now, paying attention to some notation adjustments, we can express global spacetime transformations as

$$
x^{\prime \mu}=\chi_{v}^{\mu} x^{v}+a^{\mu}
$$

which corresponds to Lorenz plus translations. Accordingly previous explanation, local implementation, however, requires at each point of spacetime

$$
x^{\prime \mu}=\chi_{v}^{\mu}(x) x^{v}+a^{\mu}(x)
$$

that is,

$$
d x^{\prime \mu}=\chi_{v}^{\mu}(x) d x^{v} .
$$

The invariance of the geodesic arc element (or the coordinate invariance of derivatives) is gained by the introduction of a new metric tensor field

$$
d s^{2}=g_{\mu \nu}(x) d x^{\mu} d x^{v}=g_{\mu \nu}^{\prime}(x) d x^{\prime \mu} d x^{\prime \nu},
$$

which transforms in accordance to

$$
g_{\gamma \eta}^{\prime}\left(x^{\prime}\right)=\chi_{\gamma}^{-1 \mu}(x) g_{\mu \nu}(x) \chi_{\eta}^{-1 v}(x) .
$$

To preserve the homogeneity of tensor transformations, the covariant derivative must obey

$$
D_{\mu} A^{\alpha} \rightarrow D_{\mu}^{\prime} A^{\alpha}=\chi_{\mu}^{v} \chi_{\beta}^{\alpha} D_{v} A^{\beta} .
$$

This condition may be achieved by

$$
D_{\mu} A^{\alpha}=\partial_{\mu} A^{\alpha}+\Gamma_{\mu \sigma}^{\alpha} A^{\sigma},
$$

with the connection

$$
\Gamma_{\mu \sigma}^{\alpha}=\frac{1}{2} g^{\alpha \rho}\left[\partial_{\mu} g_{\sigma \rho}+\partial_{\sigma} g_{\mu \rho}-\partial_{\rho} g_{\mu \sigma}\right] .
$$

Despite all this, the gauge of gravitation is entirely based on the geometric concept of the gravitational field, which derives directly from the spacetime structure, unlike the other physical fields.

Now, it is important to note that much of the essential texts on gauge theory remained in German for a long time, and some are still hardly found in translated versions, a fact that has impeded the full access to many documents by the majority of the scientific community not familiarized with the 
language [12]. For this reason, and also because of its distinct theoretical structure, unfortunately Lyra's gauge approach is not commonly treated in the general context of gauge theories, but the cogency of the insights contained in it cannot be ignored at all, especially in modern quantum cosmology.

As Lyra [13] himself said, “[...] Es besteht eine so nahe innere Verwandtschaft des hier gegebenen Aufbaus der Infinitesimalgeometrie mit demjenigen Weyls aus dem Jahre 1918, dass man ebensogut von einer Modifikation der Weylschen Geometrie sprechen könnte" (There is such a close inner relationship of the infinitesimal structure given here with that from Weyl (1918) that one could just speak of a modification of Weyl's geometry) ${ }^{1}$. Thus, Lyra's geometry is a generalization of Riemannian geometry-initially taken in a manifold not endowed of a metric - with a positive definite function, the scalar field $\chi\left(x^{k}\right)$ for scale changes, in which the reference system is defined not only by the coordinates but also by including that scalar field, that is, the gauge function $\chi\left(x^{k}\right)[13]^{2}$, so that the Levi-Civita-Christofell connection is $\chi^{-1}$-gauged and added of a negative term referring to the vector displacement of a given parallel transport between two neighboring points. Therefore, a change in reference system is in fact a change of coordinates and a gauge transformation, all at once.

A tensor metric $g_{\mu \nu}$ is subsequently introduced, and the new asymmetric connection is given by

$$
{ }^{\dagger} \Gamma_{\mu \sigma}^{\alpha}=\chi^{-1} \Gamma_{\mu \sigma}^{\alpha}-\frac{1}{2}\left(\delta_{\mu}^{\alpha} \phi_{\sigma}+\delta_{\sigma}^{\alpha} \phi_{\mu}-g_{\mu \sigma} \phi^{\alpha}\right),
$$

where ${ }^{\dagger} \Gamma_{\mu \sigma}^{\alpha}$ is symmetric in only the lower indices, ${ }^{\dagger} \Gamma_{\mu \sigma}^{\alpha}$ is the usual connection ${ }^{3}$, and $\phi_{\sigma}$ is the

1 Translated from German by the author.

2 In words from Lyra: "[...] Dabei wird der Eichbegriff nicht mehr als Festlegung von Längeneinheiten verstanden, sondern schon im strukturlosen Raum als ein mit dem Koordinatensystem gleichberechtigter Bestandteil des Bezugssystems eingeführt" (Here, the calibration term is no longer understood as establishing length units, but introduced already in the structureless space on an equal footing with the coordinate system part of the reference system). Translated from German by the author.

${ }^{3}$ Whenever possible, it is desirable to reflect upon the precise meaning of the objects under study. Weaving formal displacement vector field. The geodetic arc element in Lyra's manifold has the form

$$
d s^{2}=\chi^{2} g_{\mu \sigma} d x^{\mu} d x^{\sigma}
$$

and the change from a reference frame $\left(\chi, x^{i}\right)$ to $\left(\chi^{\prime}, x^{\prime i}\right)$ is obtained doing

$$
\chi^{\prime}=\chi\left(\chi, x^{k}\right), x^{i}=x^{i}\left(x^{k}\right)
$$

It is important to add that the Jacobian obeys

$$
\left|\frac{\partial x^{\prime}}{\partial x^{k}}\right| \neq 0
$$

and

$$
\frac{\partial \chi^{\prime}}{\partial \chi} \neq 0
$$

Lyra's geometry has the fundamental property that the length of a vector in parallel transport does not change, in contrast with Weyl's geometry.

\section{The Quantum Spacetime in Lyra's Geometry}

The phenomenological framework I proposed aims to explain some exotic effects in the interaction of two supermassive bodies. It considers an approach on quantum gravity in which it is assumed-having in mind that any region in space is continually being expanded (or compressed), so that there are no rigid structures at all-a metric in singularity functions, making it possible to analyze any infinitesimal timelike

considerations on the structure of Riemannian manifolds, Weitzenböck [14] summarized his conclusions by saying the following: "[...] die Functionen $\Gamma_{\mu v}^{\rho}$ definieren die $<<$ infinitesimale Parallelverschiebung" der Vektoren (und damit auch die von Tensoren höherer Stufe), oder auch: die Funktionen $\Gamma_{\mu v}^{\rho}$ definieren den "affinen Zusammenhang>> der Mannigfaltigkeit" ([...] function $\Gamma_{\mu \nu}^{\rho}$ defines the "infinitesimal parallel displacement" of the vectors (and thus also of tensors of higher order), or else function $\Gamma_{\mu v}^{\rho}$ defines the "affine relation" of the manifoldness). Translated from German by the author. Thus, Weitzenböck understands function $\Gamma_{\mu v}^{\rho}$ as the analytical representation of the structural geometrical essence of a Riemannian manifold, ultimately its "holonomyness" rephrased in operational description encoded by an algorithm of parallel transport. 
element of a geodesic in a gravitational singularity. In such approach, there is no vanishing of space components of the metric tensor, although the participation of space in the geodesic path is annulled simply by choosing a value of the spacelike $x$-variable for the infinitesimal element in Macauley kets, $d\langle x-\varepsilon\rangle_{\alpha}^{2}$, with the restriction $x<\varepsilon_{\alpha}[8]$. Accordingly, the space still exists in the singularity, however, as it was "frozen". This means that the geometry of spacetime fluctuates (or undergoes excitations) over "non-space", apart from the trivial case of the $g_{\mu \sigma}=0$ solution [8].

From the above scenario, since no effective displacement occurs, field becomes static in space, so that the connection

$$
\Gamma_{00}^{\alpha}=\frac{1}{2} g^{\alpha \rho}\left(\partial_{0} g_{\rho 0}+\partial_{0} g_{0 \rho}-\partial_{\rho} g_{00}\right)
$$

reduces to

$$
\Gamma_{00}^{\alpha}=\frac{1}{2} g^{\alpha \rho}\left(\partial_{0} g_{\rho 0}+\partial_{0} g_{0 \rho}\right)
$$

in which

$$
\begin{aligned}
& \partial_{0} g_{\rho 0}=\frac{\partial g_{\rho 0}}{\partial\left\langle t-\varepsilon_{0}\right\rangle}=\frac{\partial g_{\rho 0}}{\partial\langle x-\varepsilon\rangle_{0}} \\
& \partial_{\rho} g_{00}=\frac{\partial g_{00}}{\partial\left\langle x-\varepsilon_{\rho}\right\rangle}=\frac{\partial g_{00}}{\partial\langle x-\varepsilon\rangle_{\rho}} .
\end{aligned}
$$

The quantum spacetime was matched with quantum Riemannian metric in order to obtain the correlation function

$$
\left\langle 0\left|g_{\mu \sigma} d\langle x-\varepsilon\rangle_{\mu} d\langle x-\varepsilon\rangle_{\sigma}\right| 0\right\rangle=-d\langle x-\varepsilon\rangle_{0}^{2}
$$

In short, we can only predict the expectation value of the rate in which the invariant element evolves in time mode, once a G-closure is manifested. As already emphasized, such considerations were made matching quantum spacetime and quantum Riemannian metric within a unique geometric framework as a way to quantize not the gravitational field, but the spacetime on its own, establishing compliance between quantum gravity and Einstein's general relativity.
Conducting formal developments supported by some considerations of Woodard [15, 16], Rovelli [2, 4], and from the general theory of singularity functions, I concluded that, given the energy density, the expectation value of the rate at which the invariant element evolves only in time mode, within a G-closure [8], in a locally flat background, is described by

$$
\begin{aligned}
& \left\langle 0\left|g_{\mu \sigma} d\langle x-\varepsilon\rangle_{\mu} d\langle x-\varepsilon\rangle_{\sigma}\right| 0\right\rangle=\Omega^{2}\left\{-[1-C(u)] d u^{2}\right\}= \\
& =-\left[\frac{3}{8 \pi G u^{2} \rho_{\langle t-\varepsilon\rangle_{0}}}\left(1-\frac{1}{2} \frac{u}{1+A(u)} \frac{d A(u)}{d u}\right)^{2}\right] d u^{2},(13-\mathrm{a})
\end{aligned}
$$

since

$$
\frac{3 H^{2}}{8 \pi G \rho_{\left\langle t-\varepsilon_{0}\right\rangle}}\left[1-\frac{1}{2} \frac{u}{1+A(u)} \frac{d A(u)}{d u}\right]^{2}=1-C(u)
$$

where $u$ is a time function that corresponds to $1 / H$ for time coordinate equal to 0 and to 0 for time coordinate equal to $\infty, A(u)$ and $C(u)$ are functions freely evaluated non-perturbatively from the retarded Green's functions of the massless minimally coupled and conformally coupled scalars. If we take the approach from loop quantum gravity, maintaining conditions of isotropy and homogeneity previously assumed, Friedmann equation becomes

$$
\left(\frac{\dot{R}}{R}\right)^{2}=\frac{8 \pi G}{3}\left\langle\rho_{\langle t-\varepsilon\rangle_{0}}\right\rangle\left(1-\frac{\left\langle\rho_{\langle t-\varepsilon\rangle_{0}}\right\rangle}{\rho_{P l}}\right),
$$

where $\rho_{P l} \sim 10^{96} \mathrm{Kg} / \mathrm{m}^{3}$ is the Planck density [3, 4].

Although it has been produced a certain number of works applying Lyra's geometry, very little effectively was gained so far, except, perhaps, the interpretation related to the cosmological constant as I shall discuss below. Nevertheless, the search for a suitable physics to describe gravitational singularities led me to a complex geometry resulting from a combination of Lyra's geometry with the geometry of singularity functions described in Ref. [8]. Using Lyra's geometry, the gauged connection gets the general form of Eq. (8), which restricted to timelike singularity coordinates gives 


$$
{ }^{\dagger} \Gamma_{00}^{\alpha}=\frac{1}{2} \chi^{-1} g^{\alpha \rho}\left(\partial_{0} g_{\rho 0}+\partial_{0} g_{0 \rho}\right)-\frac{1}{2}\left(\delta_{0}^{\alpha} \phi_{0}+\delta_{0}^{\alpha} \phi_{0}\right),
$$

with

$$
\begin{aligned}
& \phi^{0}=\phi_{0}=\beta_{\left\langle t-\varepsilon_{0}\right\rangle},{ }^{4} \\
& \phi^{\alpha}=\phi_{\alpha}=\phi_{\langle x-\varepsilon\rangle_{\alpha}},
\end{aligned}
$$

and

$$
\phi_{\langle x-\varepsilon\rangle_{\alpha}}=0
$$

for $x<\varepsilon_{\alpha}$.

The argument that the indices simplify the formalism is really a scam. Therefore, unlike the literature in general, I shall make a careful explanation of the meaning of these expressions. As stated, $\Gamma_{\mu \sigma}^{\alpha}$ is symmetric only in lower indices, which means that " $\alpha$ " does not commute, in general, with indices " $\mu$ " and " $\sigma$ ", appearing as superscript symbolizing contravariance, i.e., infinitesimal displacement. Also, in accordance with previous deductions that led to the geodesic equation in singularity functions [8], indices " $\mu$ " and " $\sigma$ " were taken as time-labels while " $\alpha$ " and " $\rho$ " became space-labels (" $\rho$ " replaces " $\alpha$ " to characterize the metric tensor component as a function of time and space in partial derivatives, but this is done without any loss of generality). So, according to the second term in the right-hand side of Eq. (14), those infinitesimal displacements run over time, on the temporal component of the vector field, in the spatial directions " $\alpha$ " of this field. However, as there is no spatial displacement (see properties of singularity functions, taking care not to confuse "spatial direction" with "spatial displacement"), the spatial components of the displacement vector field cancel out, thus leaving Eq. (14).

\footnotetext{
4 In my previous work [8], the adoption of singularity functions aimed to allow disregard the participation of space in the calculation of the invariant commoving timelike element, with no need to guess lack of space. Thus, timelike geodesics are determined by application of the properties of Macauley kets on their space parts, since the usual differential coordinates were replaced by differentials of intervals. Thus,

$\phi_{\mu}=\left(\beta_{\langle t-\varepsilon\rangle_{0}},\langle x-\varepsilon\rangle_{1},\langle x-\varepsilon\rangle_{2},\langle x-\varepsilon\rangle_{3}\right) \rightarrow(\beta, 0,0,0)$ for $x_{\mu}<\varepsilon_{\mu}$.
}

The parallel transfer of a vector $\omega$ in Lyra's geometry is given by

$$
\delta \omega^{\alpha}=-\left({ }^{\dagger} \Gamma_{\mu \sigma}^{\alpha}-\frac{1}{2} \delta_{\mu}^{\alpha} \phi_{\sigma}\right) \omega^{\mu} \chi d x^{\sigma} .
$$

If one assumes the natural gauge $(\chi=1)$, the vector length is not changed under parallel transfer.

In its general form, the geodesic is now described by

$$
\begin{aligned}
& \chi \frac{d^{2}\langle x-\varepsilon\rangle_{\alpha}}{d \tau^{2}}+\Gamma_{\mu \sigma}^{\alpha} \frac{\chi d\langle x-\varepsilon\rangle_{\mu}}{d \tau} \frac{\chi d\langle x-\varepsilon\rangle_{\sigma}}{d \tau}=0 ; \quad(16) \\
& \frac{d^{2}\langle x-\varepsilon\rangle_{\alpha}}{d \tau^{2}}+\left[\chi^{-1} \Gamma_{\mu \sigma}^{\alpha}-\frac{1}{2}\left(\delta_{\mu}^{\alpha} \phi_{\sigma}+\delta_{\sigma}^{\alpha} \phi_{\mu}-g_{\mu \sigma} \phi^{\alpha}\right)\right] \\
& \times \frac{d\langle x-\varepsilon\rangle_{\mu}}{d \tau} \chi \frac{d\langle x-\varepsilon\rangle_{\sigma}}{d \tau}=0 ; \\
& \frac{d^{2}\langle x-\varepsilon\rangle_{\alpha}}{d \tau^{2}}+\Gamma_{\mu \sigma}^{\alpha} \frac{d\langle x-\varepsilon\rangle_{\mu}}{d \tau} \frac{d x-\varepsilon\rangle_{\sigma}}{d \tau} \\
& -\frac{\chi}{2}\left(\delta_{\mu}^{\alpha} \phi_{\sigma}+\delta_{\sigma}^{\alpha} \phi_{\mu}-g_{\mu \sigma} \phi^{\alpha}\right) \frac{d\langle x-\varepsilon\rangle_{\mu}}{d \tau} \frac{d\langle x-\varepsilon\rangle_{\sigma}}{d \tau}=0 .
\end{aligned}
$$

Lastly, for timelike geodesics in singularity representation,

$$
\frac{d^{2}\langle x-\varepsilon\rangle_{\alpha}}{d \tau^{2}}+\Gamma_{00}^{\alpha} \frac{d\langle x-\varepsilon\rangle_{0}}{d \tau} \frac{d\langle x-\varepsilon\rangle_{0}}{d \tau}
$$

$-\frac{\chi}{2}\left(\delta_{0}^{\alpha} \phi_{0}+\delta_{0}^{\alpha} \phi_{0}\right) \frac{d\langle x-\varepsilon\rangle_{0}}{d \tau} \frac{d\langle x-\varepsilon\rangle_{0}}{d \tau}=0$.

An obvious advantage of Lyra's geometry is that under the new connection a vector length is unchanged after a parallel transfer, which is physically appropriate, especially in the case of displacements only in time, whose comprehension is far from trivial. Also, as yet we'll see below, Lyra's geometry has raised new interpretations to the cosmological constant from Einstein's equations.

We consider, for instance, the FLRW background. As we know, Einstein's field equation in Lyra geometry is 


$$
G_{\mu \sigma}+\frac{3}{2} \phi^{\mu} \phi_{\sigma}-\frac{3}{4} \delta_{\mu \sigma} \phi^{\alpha} \phi_{\alpha}=-\kappa T_{\mu \sigma},
$$

which gives

$$
\begin{aligned}
G_{00}+\frac{3}{2} \phi^{0} \phi_{0}-\frac{3}{4} \delta_{00} \phi^{0} \phi_{0} & =-\kappa T_{00} \\
G_{11}+\frac{3}{2} \phi^{1} \phi_{1}-\frac{3}{4} \delta_{11} \phi^{1} \phi_{1} & =-\kappa T_{11} ; \\
G_{22}+\frac{3}{2} \phi^{2} \phi_{2}-\frac{3}{4} \delta_{22} \phi^{2} \phi_{2} & =-\kappa T_{22} ; \\
G_{33}+\frac{3}{2} \phi^{3} \phi_{3}-\frac{3}{4} \delta_{33} \phi^{3} \phi_{3} & =-\kappa T_{33} .
\end{aligned}
$$

Restricted to timelike geodesics, as in the quantum theory of spacetime presented in Ref. [8], we stay with

$$
G_{00}+\frac{3}{2} \phi^{0} \phi_{0}-\frac{3}{4} \delta_{00} \phi^{0} \phi_{0}=-\kappa T_{00} \text {. }
$$

From this, the Friedmann-like field equation is written as

$$
G_{00}+\frac{3}{4} \delta_{00} \beta^{2}=-\kappa T_{00}
$$

or

$$
-G_{00}-\frac{3}{4} \delta_{00} \beta^{2}=\kappa T_{00}
$$

Since we have

$$
-G_{00}=\frac{3 k}{R^{2}}+3\left(\frac{\dot{R}}{R}\right)^{2},
$$

then

$$
\begin{gathered}
3\left(\frac{\dot{R}}{R}\right)^{2}+\frac{3 k}{R^{2}}-\frac{3}{4} \beta_{\langle t-\varepsilon\rangle_{0}}^{2}=\kappa \rho_{\langle t-\varepsilon\rangle_{0}}, \\
\left(\frac{\dot{R}}{R}\right)^{2}+\frac{k}{R^{2}}-\frac{1}{4} \beta_{\langle t-\varepsilon\rangle_{0}}^{2}=\frac{8 \pi G}{3} \rho_{\langle t-\varepsilon\rangle_{0}} .
\end{gathered}
$$

Confronting this equation with Eq. (13-c), we see that it is valid when the matter in the Universe has a density negligible compared to the Planck density (the parenthesis in Eq. (13-c) is close to 1, and correction is insignificant). All the letters designate the well known quantities of GR and cosmology, unless otherwise indicated. Nowadays, many authors understand the constant displacement vector field in Lyra formalism with the same physical role as the cosmological constant in the standard GR. In this sense, we can say that the cosmological constant naturally results from the introduction of the Lyra gauge. Therefore, it is expected the new gauge could reflect the characteristics of the cosmological constant term, that is

$$
\phi^{1} \phi_{1}=\phi^{2} \phi_{2}=\phi^{3} \phi_{3}
$$

\section{Discussion and Conclusion}

Timelike geodesics are treated with a bit of common sense even if one understands that it is in a conceptual level very far from a naive physical framework. This is so because time in GR is not the time of clocks but an evolutionary variable, and it is difficult to us to discard old archetypes like rules and clocks.

Whenever we seek a new physics to give account for an almost impenetrable phenomenon we try to find the invariants of the theory, the referents that make possible to get some knowledge about, and this search unwittingly drags us again to the classical measuring tools for thought experiments. From my point of view, the most interesting thing about the introduction of Lyra's gauge is the feasibility of the description of a notional parallel transfer in time without changing the duration, regardless of the spatial direction. This is an invariant useful to describe one of the quantum faces of gravity and goes far beyond common sense.

As we have seen briefly, physicists try to interpret the real meaning of Lyra's extra-displacement terms in Einstein's equations giving to them the role of cosmological constant. Nevertheless, in my approach we have to return to Lyra's geometry discussing what is a time parallel transfer of a time interval in a certain direction. I remember that space is "frozen" in the singularity representation of a timelike geodesic; there is no space displacement. Therefore, in the natural gauge a time parallel transfer of a time interval is in fact a projection of this time interval in one space direction targeting another virtual geodesic path in which space 
coordinates would be also treated by singularity functions. This is a way to say that, under the same conditions, we have the same behavior of nature. In my work, these conditions feature the so-called G-closure. Importantly, this geometric review in no way precludes the representation of the cosmological constant; rather, it emphasizes the invariance of the duration under parallel transport, thus characterizing a constancy of nature.

Lastly, comparing quantum spacetime with quantum Riemannian metric to establish the conditions for further precise evaluation of the functions $A(u)$ and $C(u)$ in present model (expressions 13-a and 13-b), we in fact are asking what functions $A(u)$ and $C(u)$ relate quantum spacetime with quantum Riemannian metric, measuring the spacetime shrinkage rate. So, we can say that the geometry of spacetime fluctuates and this is the same to say that Riemannian metric fluctuates as the physical state of spacetime itself.

\section{Acknowledgements}

The author would like to thank Professor Dr. José Abdalla Helayël Neto, at Brazilian Center for Physics Research, old friend and research partner, for the support and the always important observations (josehelayel@gmail.com). The author also thanks Professor Antonio Fernandes da Fonseca Teixeira, at Brazilian Center for Physics Research, by the time generously dedicated to some important debates (teixeira@cbpf.br).

\section{References}

[1] Rovelli, C. 2006. "The Disappearance of Space and Time." In The Ontology of Spacetime, Philosophy of
Physics, edited by Dieks, D. Elsevier Science Publishing Co., 25.

[2] Rovelli, C. 2007. "Quantum Gravity." In Philosophy of Physics, edited by Butterfield, J. and Earman, J. Elsevier Science Publishing Co., 1287.

[3] Rovelli, C. 2007. "Beyond the Screen of Time." Nature Physics 3.

[4] Rovelli, C. 2011. "Zakopane Lectures on Loop Gravity." arXiv: 1102.3660 .

[5] Rovelli, C. 2012. Et si Le Temps n'Existait Pas? Un Peu de Science Subversive. Paris : Dunod.

[6] Baez, J. 2005. "Quantum Quandaries: A Category-Theoretic Perspective." In Structural Foundations of Quantum Gravity, edited by Rickles, D., French, S., and Saatsi, J. Oxford University Press.

[7] Butterfield, J., and Isham, C. 2000. "Spacetime and the Philosophical Challenge of Quantum Gravity." In Physics Meets Philosophy at the Planck Scale. Cambridge University Press.

[8] Serpa, N., and Steiner R. 2016. "General Relativity, Quantum Gravity and All That: Time Machines in Perspective by Singularity Functions." Bulg. Jour. Phys. 43 (1): 1-20.

[9] Reddy, D., and Innaiah, P. 1986. Astrophys. Space Sci. 123-49.

[10] Reddy, D., and Venkateswarlu, R. 1987. Astrophys. Space Sci. 136-83.

[11] Shchigolev, V. 2013. "On Exact Cosmological Models of a Scalar Field in Lyra Geometry." Universal Journal of Physics and Application 1(4): 408-13.

[12] O'Raifeartaigh, L. 1997. The Dawning of Gauge Theory. New Jersey: Princeton University Press.

[13] Lyra, G. 1951. "Über eine Modifikation der Riemannschen Geometrie." Mathematische Zeitschrift 54 (1): 52-64.

[14] Weitzenböck, R. 1923. Invariantentheorie. Groningen: Noordhoff.

[15] Woodard, R. 1995. "Results from Quantum Cosmological Gravity." In Unified Symmetry: In the Small and in the Large. New York: Plenum Press.

[16] Woodard, R. 2009. "How Far Are We from the Quantum Theory of Gravity?” arXiv: 0907.4238. 